\title{
Breast-Cancer Predisposition in Multiple Endocrine Neoplasia Type 1
}

TO THE EDITOR: Multiple endocrine neoplasia type 1 (MEN1) is caused by germline mutations in the MEN1 tumor-suppressor gene and is typically characterized by parathyroid adenomas, duodenopancreatic neuroendocrine tumors, and pituitary adenomas. ${ }^{1}$ Recent studies in animals ${ }^{2}$ suggest that MEN1 is involved in breast-cancer initiation. Through its encoding of menin, a coregulator of estrogen receptor $\alpha$, MEN1 has been implicated in breast-cancer progression. ${ }^{3,4}$

To clarify the role of MEN1 in human breast cancer, the International Breast Cancer in MEN1 Study Group assessed the incidence of breast cancer in the Dutch longitudinal MEN1 database, which includes more than $90 \%$ of Dutch patients with MEN1. ${ }^{5}$ (Study group members are listed in the Supplementary Appendix, available with the full text of this letter at NEJM.org.) In 190 female patients with MEN1, the relative risk of invasive breast cancer was $2.83(\mathrm{P}<0.001)$, with a standardized incidence ratio of 2.14 (95\% confidence interval [CI], 1.18 to 3.86) (Table S1 in the Supplementary Appendix). The mean $( \pm S D)$ age at diagnosis of mostly luminal-type breast cancer was $48.0 \pm 8.8$ years, as compared with an age of 60 to 65 years in the general population. Three of the 12 Dutch breast-cancer patients with MEN1 had a history of hyperprolactinemia. Risk ratios for other major cancers were not elevated (Table S1 in the Supplementary Appendix).

We validated the observations in three independent cohorts from the United States, Tasmania, and France that included a total of 675 female patients with MEN1. In a comparison of breast-cancer prevalence and incidence with corresponding data from the respective national cancer registries, the risk ratios were 2.40 in the United States $(\mathrm{P}=0.11), 2.31$ in Tasmania $(\mathrm{P}=0.22)$, and 2.33 in France $(P=0.03)$. (The comparisons were not significant in the United States and Tasmania because of the small numbers of patients in these cohorts.) The standardized incidence ratio in the combined verification cohorts was 1.96 (95\% CI, 1.33 to 2.88), and the average age at diagnosis was 51 years.

Nuclear localization of menin was reduced by more than $50 \%$ in 8 of 10 breast-cancer samples obtained from Dutch patients with MEN1 on immunohistochemical staining with the use of menin antibody A300-105A (Bethyl Laboratories) (Table 1). Subsequent analysis on DNA sequencing or multiplex ligation-dependent probe amplification revealed loss of heterozygosity at the MEN1 locus in 3 of 9 tumors. Reduced menin

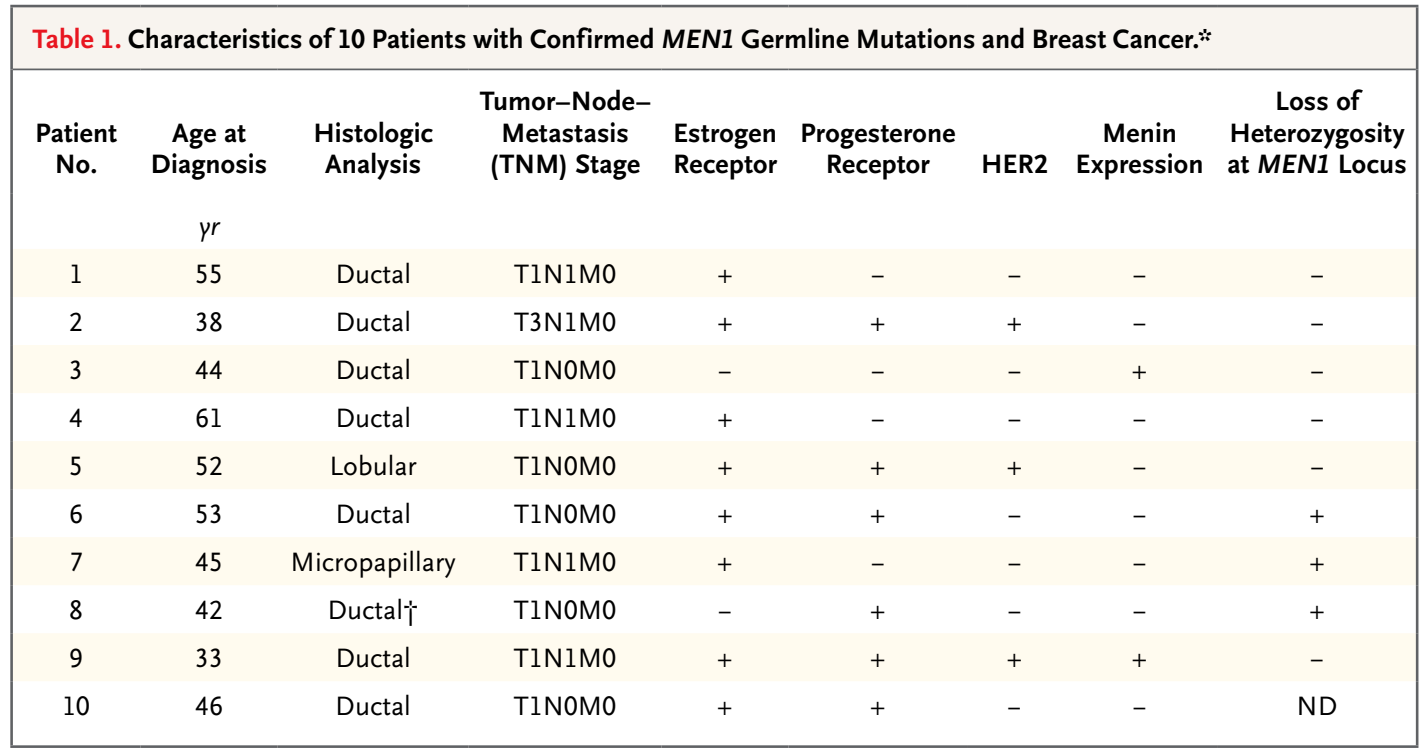

* A plus sign indicates positivity, and a minus sign negativity. HER2 denotes human epidermal growth factor receptor 2 , and ND not determined.

$\dagger$ This patient had a tumor in each breast. 
staining was found in only 4 of 88 control breast tumors on a tissue microarray $(\mathrm{P}<0.001$ by Pearson's chi-square test). All studies were conducted in accordance with regulations of the local institutional review board.

In conclusion, female patients with MEN1 are at increased risk for breast cancer. Loss of menin expression and loss of heterozygosity at the MEN1 locus in a subgroup of patients suggest a mammary-cell autonomous effect in MEN1-related breast cancer. Our observations indicate that MEN1 mutations are involved in human breast carcinogenesis. Further research will clarify the relevance of MEN1 function for patients with nonfamilial breast cancer. Intensified breastcancer screening at a relatively young age should be considered in female patients with MEN1.

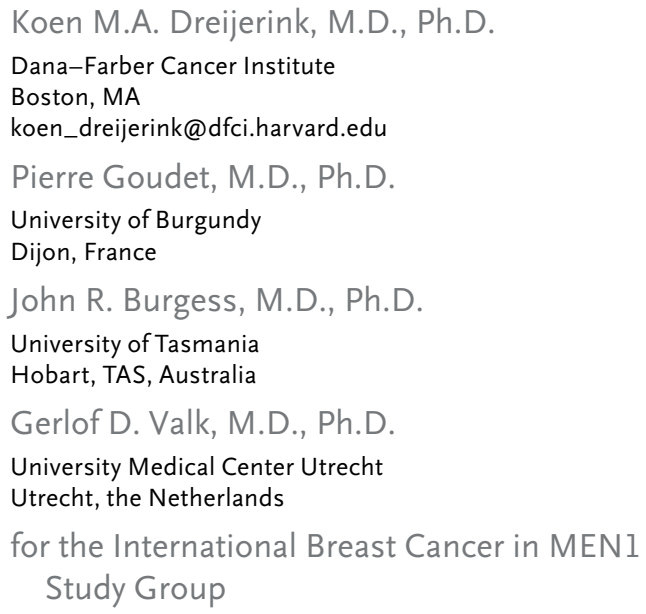

Supported by grants from the Dutch Cancer Society (to Dr. Dreijerink), Ipsen Pharmaceuticals, and the Comprehensive Cancer Center of the Netherlands (to Dr. Valk).

Disclosure forms provided by the authors are available with the full text of this letter at NEJM.org.

1. Thakker RV, Newey PJ, Walls GV, et al. Clinical practice guidelines for multiple endocrine neoplasia type 1 (MEN1). J Clin Endocrinol Metab 2012;97:2990-3011.

2. Seigne C, Auret M, Treilleux I, et al. High incidence of mammary intraepithelial neoplasia development in Men1-disrupted murine mammary glands. J Pathol 2013;229:546-58.

3. Imachi $\mathrm{H}$, Murao $\mathrm{K}$, Dobashi $\mathrm{H}$, et al. Menin, a product of the MENI gene, binds to estrogen receptor to enhance its activity in breast cancer cells: possibility of a novel predictive factor for tamoxifen resistance. Breast Cancer Res Treat 2010;122:395-407. 4. Dreijerink KM, Mulder KW, Winkler GS, Höppener JW, Lips CJ, Timmers HT. Menin links estrogen receptor activation to histone H3K4 trimethylation. Cancer Res 2006;66:4929-35.

5. de Laat JM, Pieterman CR, Weijmans M, et al. Low accuracy of tumor markers for diagnosing pancreatic neuroendocrine tumors in multiple endocrine neoplasia type 1 patients. J Clin Endocrinol Metab 2013;98:4143-51.

DOI: 10.1056/NEJMc1406028

Correspondence Copyright (c) 2014 Massachusetts Medical Society.

\section{INSTRUCTIONS FOR LETTERS TO THE EDITOR}

Letters to the Editor are considered for publication, subject to editing and abridgment, provided they do not contain material that has been submitted or published elsewhere. Please note the following:

- Letters in reference to a Journal article must not exceed 175 words (excluding references) and must be received within 3 weeks after publication of the article.

- Letters not related to a Journal article must not exceed 400 words.

- A letter can have no more than five references and one figure or table.

- A letter can be signed by no more than three authors.

- Financial associations or other possible conflicts of interest must be disclosed. Disclosures will be published with the letters. (For authors of Journal articles who are responding to letters, we will only publish new relevant relationships that have developed since publication of the article.)

- Include your full mailing address, telephone number, fax number, and e-mail address with your letter.

- All letters must be submitted at authors.NEJM.org.

Letters that do not adhere to these instructions will not be considered. We will notify you when we have made a decision about possible publication. Letters regarding a recent Journal article may be shared with the authors of that article. We are unable to provide prepublication proofs. Submission of a letter constitutes permission for the Massachusetts Medical Society, its licensees, and its assignees to use it in the Journal's various print and electronic publications and in collections, revisions, and any other form or medium.

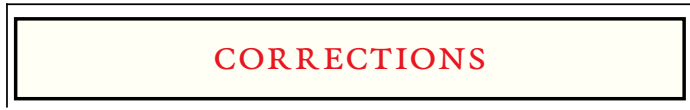

Ledipasvir and Sofosbuvir for Previously Treated HCV Genotype 1 Infection (April 17, 2014;370:1483-93). In the fourth paragraph of the Discussion (page 1491), the first sentence should have ended, ". . . the rates of response were similar in patients with cirrhosis (100\% with both regimens) and those without cirrhosis (99\% with both regimens)," rather than ". . . patients with cirrhosis (99\% with both regimens) and those without cirrhosis (100\% with both regimens)." The article is correct at NEJM.org.

Phase 3 Trials of Solanezumab for Mild-to-Moderate Alzheimer's Disease (January 23, 2014;370:311-21). In Results, in the Biologic Markers and Neuroimaging Outcomes subsection, the final sentence of the penultimate paragraph (page 318) should have begun, "Whole-brain volume decreased slightly in the solanezumab group and the placebo group ....," rather than "Whole-brain volume increased slightly . . . ." The article is correct at NEJM.org.

Upper-Airway Stimulation for Obstructive Sleep Apnea (January 9, 2014;370:139-49). In the list of authors (page 139), Dr. Vanderveken's first name should have been "Olivier," rather than "Oliver." The article is correct at NEJM.org. 